Sensors 2006, 6, 64-79

sensors

ISSN 1424-8220

(C) 2006 by MDPI

http://www.mdpi.org/sensors

Full Research Paper

\title{
Improving the Response of a Wheel Speed Sensor by Using a RLS Lattice Algorithm
}

\author{
Wilmar Hernandez \\ Department of Circuits and Systems in the EUIT de Telecomunicación at the Universidad Politécnica \\ de Madrid (UPM), Campus Sur UPM, Ctra. Valencia km 7, Madrid 28031, Spain \\ Phone: +34913367830. Fax:+34913367829. E-mail: whernan@ics.upm.es
}

Received: 3 February 2006 / Accepted: 23 February 2006 / Published: 23 February 2006

\begin{abstract}
Among the complete family of sensors for automotive safety, consumer and industrial application, speed sensors stand out as one of the most important. Actually, speed sensors have the diversity to be used in a broad range of applications. In today's automotive industry, such sensors are used in the antilock braking system, the traction control system and the electronic stability program. Also, typical applications are cam and crank shaft position/speed and wheel and turbo shaft speed measurement. In addition, they are used to control a variety of functions, including fuel injection, ignition timing in engines, and so on. However, some types of speed sensors cannot respond to very low speeds for different reasons. What is more, the main reason why such sensors are not good at detecting very low speeds is that they are more susceptible to noise when the speed of the target is low. In short, they suffer from noise and generally only work at medium to high speeds. This is one of the drawbacks of the inductive (magnetic reluctance) speed sensors and is the case under study. Furthermore, there are other speed sensors like the differential Hall Effect sensors that are relatively immune to interference and noise, but they cannot detect static fields. This limits their operations to speeds which give a switching frequency greater than a minimum operating frequency. In short, this research is focused on improving the performance of a variable reluctance speed sensor placed in a car under performance tests by using a recursive least-squares (RLS) lattice algorithm. Such an algorithm is situated in an adaptive noise canceller and carries out an optimal estimation of the relevant signal coming from the sensor, which is buried in a broad-band noise background where we have little knowledge of the noise characteristics. The experimental results are satisfactory and show a significant improvement in the signal-to-noise ratio at the system output.
\end{abstract}


Keywords: wheel speed sensor; adaptive noise canceller; recursive least-squares lattice adaptive filter

\section{Introduction}

The automobile is one of our main means of transportation, and we make extensive use of it throughout our lives. This reason, among others, justifies the necessity of the application of the advances in sensors, instrumentation, estimation and control, etc., in building cars able to make intelligent driving decisions with the objective of improving our road safety.

In addition, in order to guarantee an effective and reliable performance of the electronic systems of today's cars, the correct choice and location of the electronic devices and the optimal signal processing of the information coming from them are essential.

The reality is that the continuously growing need for better comfort and safety makes it almost impossible to imagine a future without intelligent systems looking after us. This is why, in the last decades, researchers from all around the world have been working hard to invent intelligent devices consisting of not only sensors, but also advanced materials [1] and microprocessors, among other devices, that incorporate a certain amount of intelligence to the sensors themselves, transforming them into better prepared measuring systems [2-25].

However, the process of fabricating sensors is not an easy task. Each application has its own requirements that make the same sensor suitable for some applications and unsuitable for others. This is way some industries impose tougher restrictions on the manufacturing of sensors than do others. Some of the strictest standard can be found in the automotive industry. There, the sensors have to work under severe working conditions such as the endurance of high temperatures, high humidity, chemical attacks, undesirably strong vibrations, electromagnetic interference, pollution, and so on. In short, the challenge that the sensors in automotive technology face is huge and very complex.

This paper shows the improvement of the real-time response of a wheel speed sensor placed in a car under performance tests. In this case, we have to deal with disturbances and/or interferences whose characteristics we have little knowledge of, and we use efficient methods of estimation in order to get clear information about the physical magnitude or process that we want to measure. Furthermore, due to the fact that the signal of interest and the noise share a similar frequency band, it is very difficult to eliminate the interference by using the classical approach to filtering, and the background noise causes serious difficulties. Therefore, in order to get the best estimation of the corrupted signal, an optimal adaptive noise canceller is used, obtaining satisfactory results.

\section{The wheel speed sensor}

The speed of rotation of the wheels is among the most important inputs to the optimal braking system of the car. In addition, other uses of the information from the rotational speed of the car's wheel include: traction control, vehicle stability control, transmission control, engine management, chassis control, hill-holder brakes, rollback detection or electronic parking brakes, brake-force distribution and roll-over protection, among others. 
In this paper, a proximity sensor held in a protective casing and mounted in a fixed position close to one of the wheels of the car undergoing performance tests was used. The proximity sensor is of the variable reluctance type and its coil is made of a thin wire wounded around an insulating form and coupled to a permanent magnet.

For the kind of tests carried out in this work, this device was only used to measure the rotation of the wheels of the car; however, in the process industries this kind of sensor has lots of applications in measuring rotation, position and location.

\subsection{Principles}

When the proximity sensor detects the presence of any of the ferrous teeth of a toothed wheel, an output voltage is obtained (see Fig. 1) because the ferrous teeth cross the magnetic field that is created in front of the sensor, causing a change in the resulting flow and producing an electromotive force in the coil. Thus, the output is an alternating signal whose frequency and amplitude are both proportional to the speed of rotation. A block diagram representing the measurement system is shown in Fig. 2.

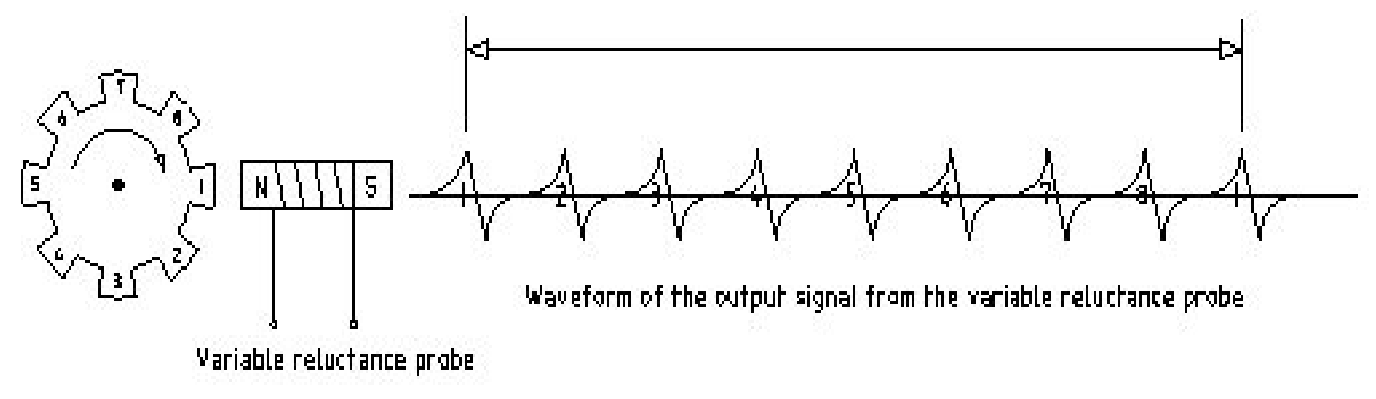

Figure 1. The sensor.

Variable геluctance ргохіmity эепsог

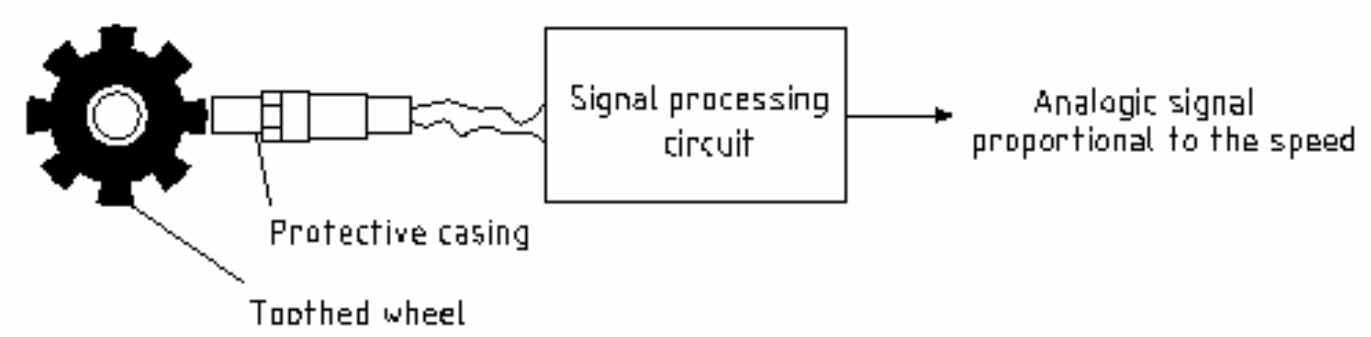

Figure 2. Block diagram of the measurement system. 


\subsection{Considerations}

In spite of the fact that variable reluctance proximity sensors are widely used in many industrial applications [26-39], it is important to point out that such sensors have advantages and disadvantages that should be considered before using them to improve the performance of today's cars.

On the one hand, they can be very small and we can embed them in places where other sensors may not fit. In addition, they are often sealed in protective cases and can be resistant to dangerous chemical attacks, high temperatures and high pressures. Also, they can detect ferromagnetic materials up to 2.5 $\mathrm{mm}$ away and they require no external power.

Furthermore, other advantages are their flexibility, reliability, small size, the little maintenance required, and low cost.

On the other hand, they should be placed very close to a suitable ferrous metal to produce an adequate output voltage. They also suffer from undesirable signals or noise, and generally only work at medium to high speed. In fact, the zero speed sensing is impossible using passive sensing technology. The traction control system, the vehicle stability control system, the anti-lock braking system (ABS) and the adaptive cruise control system are examples of applications where sensing at near zero is required. What is more, for traction control and ABS systems, sensing at near zero speed, i.e. below 7 $\mathrm{km} / \mathrm{h}$, is very important. However, unfortunately, variable reluctance proximity sensors are very susceptible to noise at automobile speeds lower than $5 \mathrm{~km} / \mathrm{h}$.

On balance, despite the fact that there are disadvantages, variable reluctance proximity sensors seem to be the most suitable choice to measure the speed of rotation of motor car wheels in the ABS of today's automobiles. For this reason, today's researchers are working hard to overcome the drawbacks of such sensors inventing new devices consisting of not only the sensors, but also signal conditioning electronics, digital signal processors, microcontrollers and field-programmable gate arrays, among other devices, that allow the sensors to make intelligent decisions that can save thousands of lives each year in car accidents.

\section{The most important sources of disturbances and noise that corrupt the relevant signal coming from wheel speed sensors}

The most important sources of disturbances and noise that corrupt the relevant signal coming from wheel speed sensors when measuring the speed of cars are the following:

- Vibrations of the framework,

- Vibrations of car sprung and unsprung masses,

- Vibrations of the chassis,

- Vibrations of both the front axle and the rear axle,

- Vibrations of the engine,

- Vibrations of the wheels,

- Vibrations of the tires,

- Vibrations caused by direct actions. 
Information about the eigenfrequencies of the above vibrations can be found in [24, 40]. Furthermore, there are other noise sources such as environmental factors, poor roads and the noise generated by the car's electrical system. These noise sources are treated as random processes [41-43].

\section{Considerations on adaptive filtering}

\subsection{Introduction}

Adaptive filtering is a very important field of research that is focused on the design of selfdesigning systems with the ability to perform satisfactorily in an environment where complete knowledge of the relevant signal characteristics is not available.

The aim of adaptive filtering is, as in any other kind of filtering, to suppress the noise that corrupts the signal of interest without causing damage to the relevant signal. However, it is important to point out that adaptive filters perform much better than classical filters in applications where the unwanted information and the relevant signal share the same frequency spectrum.

As a matter of fact, the more the noise and the relevant signal share the same (or a very similar) frequency spectrum, the less the designer can remove the unwanted information by using classical filters [24, 44-49]. Furthermore, if the signal of interest and/or the noise are not stationary processes, which is also the case under study, the use of a Wiener filter [50, 51] is inadequate [24].

According to Hernandez [24], an adaptive filter is a filter with a mechanism for adjusting its own parameters automatically by using a recursive algorithm at the same time that it in active interaction with the environment. In addition, all this happens in such a way that the performance of the adaptive filter is continuously improved according to a specified performance criterion (or cost function) which has been previously established by the designer.

In addition, the choice of an algorithm over another to design an optimal adaptive filter is determined by the following factors [53-55]:

- Low computational burden,

- Good numerical behavior,

- Robustness,

- Ease of implementation,

- Satisfactory rate of convergence,

- Good round-off error rejection.

In accordance with the above statements, in the present paper, a recursive least-squares (RLS) lattice algorithm was chosen to carry out the process of optimal estimation of the relevant signal [5458]. Furthermore, the application of such an adaptive filter is an interference or noise canceller [53, 55]. Fig. 3 shows a block diagram representation of the adaptive noise canceller, and a summary of it is given in the next subsection. 


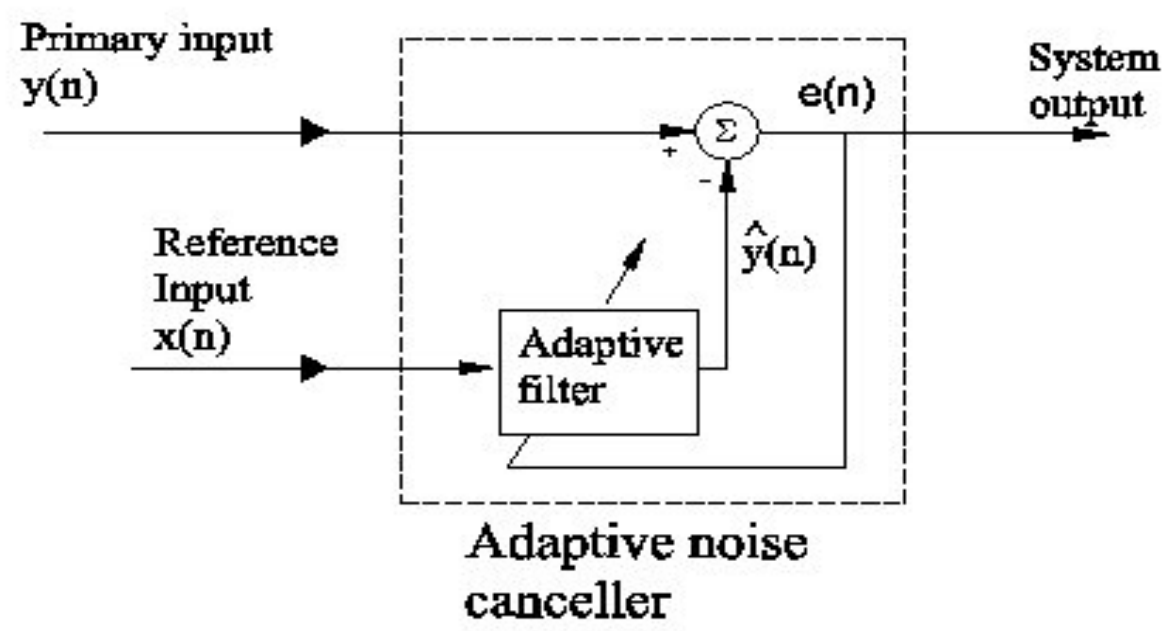

Figure 3. Block diagram representation of the adaptive filter.

\subsection{Summary of the RLS lattice algorithm (from Haykin [55] and Hernandez [24])}

According to Haykin [55], this algorithm is based on a priori estimation errors, and the reflection and joint-process estimation coefficients are all derived directly. The algorithm is called the RLS lattice algorithm using a priori estimation errors with error feedback. Additional information about the ways to derive this algorithm and its advantages and disadvantages can be found in $[24,55,56]$.

\subsubsection{The RLS lattice algorithm using a priori estimation errors with error feedback}

\subsubsection{Initialization}

To initialize the algorithm, at time $\mathrm{n}=0$, set

$$
\begin{gathered}
\Phi_{\mathrm{r}-1}(0)=\delta \\
\Theta_{\mathrm{r}-1}(-1)=\delta \\
\gamma_{\Phi, \mathrm{r}}(0)=\pi_{\Theta, \mathrm{r}}(0)=0 \\
\kappa_{0}(0)=1
\end{gathered}
$$

where $\delta$ is a small positive constant, $\Phi$ is the forward prediction-error energy, $\Theta$ is the backward prediction-error energy, $r$ is the order of the least-squares predictor and $r=1,2, \ldots, R$, where $R$ is the final order of the least-squares predictor. In addition, $\gamma$ is the forward reflection coefficient, $\pi$ is the backward reflection coefficient, and $\kappa$ is the conversion factor.

For each instant $\mathrm{n} \geq 1$, generate the zeroth-order variables:

$$
\eta_{0}(\mathrm{n})=\beta_{0}(\mathrm{n})=\mathrm{x}(\mathrm{n})
$$




$$
\begin{gathered}
\Phi_{0}(\mathrm{n})=\Theta_{0}(\mathrm{n})=\lambda \Phi_{0}(\mathrm{n}-1)+|\mathrm{x}(\mathrm{n})|^{2} \\
\kappa_{0}(\mathrm{n}-1)=1
\end{gathered}
$$

where the constant $\lambda, 0<\lambda \leq 1$, is the forgetting factor and its typical values used are the real numbers in the range from 0.99 to $1, \eta$ is the forward a priori prediction error, $\beta$ is the backward a priori prediction error, and $\mathrm{x}$ is the reference input.

For joint-process estimation, at time $\mathrm{n}=0$, set

$$
\sigma_{r-1}(0)=0
$$

At each instant $n \geq 1$, generate the zeroth-order variable

$$
\varepsilon_{0}(\mathrm{n})=\mathrm{y}(\mathrm{n})
$$

where $\sigma$ is the tap-weight vector of the transversal filter. It contains $\mathrm{R}+1$ taps. Also, $\mathrm{y}$ is the primary input and $\varepsilon$ is the system output.

\subsubsection{Predictions}

For $n=1,2,3, \ldots$, compute the various order updates in the sequence $r=1,2, \ldots, R$.

$$
\begin{gathered}
\Phi_{\mathrm{r}-1}(\mathrm{n})=\lambda \Phi_{\mathrm{r}-1}(\mathrm{n}-1)+\kappa_{\mathrm{r}-1}(\mathrm{n}-1) \mid \eta_{\mathrm{r}-1}(\mathrm{n})^{2} \\
\Theta_{\mathrm{r}-1}(\mathrm{n}-1)=\lambda \Theta_{\mathrm{r}-1}(\mathrm{n}-2)+\kappa_{\mathrm{r}-1}(\mathrm{n}-1) \mid \beta_{\mathrm{r}-1}(\mathrm{n}-1)^{2} \\
\eta_{\mathrm{r}}(\mathrm{n})=\eta_{\mathrm{r}-1}(\mathrm{n})+\gamma_{\Phi, \mathrm{r}}^{*}(\mathrm{n}-1) \beta_{\mathrm{r}-1}(\mathrm{n}-1) \\
\beta_{\mathrm{r}}(\mathrm{n})=\beta_{\mathrm{r}-1}(\mathrm{n}-1)+\pi_{\Theta, \mathrm{r}}^{*}(\mathrm{n}-1) \eta_{\mathrm{r}-1}(\mathrm{n}) \\
\gamma_{\Phi, \mathrm{r}}(\mathrm{n})=\gamma_{\Phi, \mathrm{r}}(\mathrm{n}-1)-\frac{\kappa_{\mathrm{r}-1}(\mathrm{n}-1) \beta_{\mathrm{r}-1}(\mathrm{n}-1)}{\Theta_{\mathrm{r}-1}(\mathrm{n}-1)} \eta_{\mathrm{r}}^{*}(\mathrm{n}) \\
\pi_{\Theta, \mathrm{r}}(\mathrm{n})=\pi_{\Theta, \mathrm{r}}(\mathrm{n}-1)-\frac{\kappa_{\mathrm{r}-1}(\mathrm{n}-1) \eta_{\mathrm{r}-1}(\mathrm{n})}{\Theta_{\mathrm{r}-1}(\mathrm{n}-1)} \beta_{\mathrm{r}}^{*}(\mathrm{n}) \\
\kappa_{\mathrm{r}}(\mathrm{n}-1)=\kappa_{\mathrm{r}-1}(\mathrm{n}-1)-\frac{\kappa_{\mathrm{r}-1}^{2}(\mathrm{n}-1) \beta_{\mathrm{r}-1}(\mathrm{n}-1)^{2}}{\Theta_{\mathrm{r}-1}(\mathrm{n}-1)}
\end{gathered}
$$

\subsubsection{Filtering}


For $n=1,2,3, \ldots$, compute the various order updates in the sequence $r=1,2, \ldots, R+1$ :

$$
\begin{gathered}
\varepsilon_{\mathrm{r}}(\mathrm{n})=\varepsilon_{\mathrm{r}-1}(\mathrm{n})-\sigma_{\mathrm{r}-1}^{*}(\mathrm{n}-1) \beta_{\mathrm{r}-1}(\mathrm{n}) \\
\sigma_{\mathrm{r}-1}(\mathrm{n})=\sigma_{\mathrm{r}-1}(\mathrm{n}-1)+\frac{\kappa_{\mathrm{r}-1}(\mathrm{n}) \beta_{\mathrm{r}-1}(\mathrm{n})}{\Theta_{\mathrm{r}-1}(\mathrm{n}-1)} \varepsilon_{\mathrm{r}}^{*}(\mathrm{n})
\end{gathered}
$$

where the asterisk denotes complex conjugation.

Before moving on to the results of the experiment, it is important to point out that, as stated in the initialization step, the output of the filtering process $\left(\varepsilon_{\mathrm{R}+1}\right)$ is the system output (see Fig. 3). Moreover, the outputs of the lattice predictor $\left(\eta_{R}\right.$ and $\left.\beta_{R}\right)$ are the variables used in this paper to obtain the cost function (see Section 5).

\section{Results of the experiment}

\subsection{The adaptive noise canceling system}

In the present paper, another speed sensor with the same characteristics as the wheel speed sensor and placed close to it, but far from the toothed wheel, was used to obtain information from the electrical noise of this type of sensors. In addition, a signal conditioning circuit was used to measure the electrical noise from the car battery. Moreover, an accelerometer was placed on the front axle and close to the wheel speed sensor to measure the noise generated by the mechanical vibrations.

Figure 4 shows a block diagram of the adaptive noise canceling system. Here, the transfer functions $\mathrm{H}_{1}, \mathrm{H}_{2}$ and $\mathrm{H}_{3}$ are used to show that the signals from the noise sources are uncorrelated with the relevant signal but correlated in some way with its noise. In short, these transfer functions represent the correlation between the noise that corrupts the relevant signal and the additive noise consisting of the noise coming from the second speed sensor, the noise coming from the battery of the car and the noise coming from the accelerometer. As a matter of fact, experience tells us that part of the noise of the primary input is correlated in some way with the above mentioned additive noise.

In Fig. 4, it can be seen that the reference input contains information from the additive noise corrupting the signal of interest. In addition, in such a figure the output of the adaptive filter is an estimate of the noise of the primary input. Therefore, the system output is an estimate of the relevant signal coming from the wheel speed sensor.

\subsection{Comments on the system's inputs}

Before continuing on to the next subsection, it should be stressed at this point that the frequency bands of the useful signal and the unwanted signals overlap; they are mixed with each other. First of all, the frequency band of the noise generated by the mechanical vibrations lie in the low frequency range affecting the information from the wheel speed sensor at low and medium car speed and the measurement of the tire-road coefficient of friction. In addition, the electrical noise of this type of sensor is dangerous at a very low car speed, because in those situations the power of the electrical noise of the sensor is greater than the one of the useful signal. 
Moreover, the noise generated by the electrical systems of the car and the one introduced by the electronic systems, signal conditioning circuits, hardware and data acquisition cards, are always affecting the relevant signal.

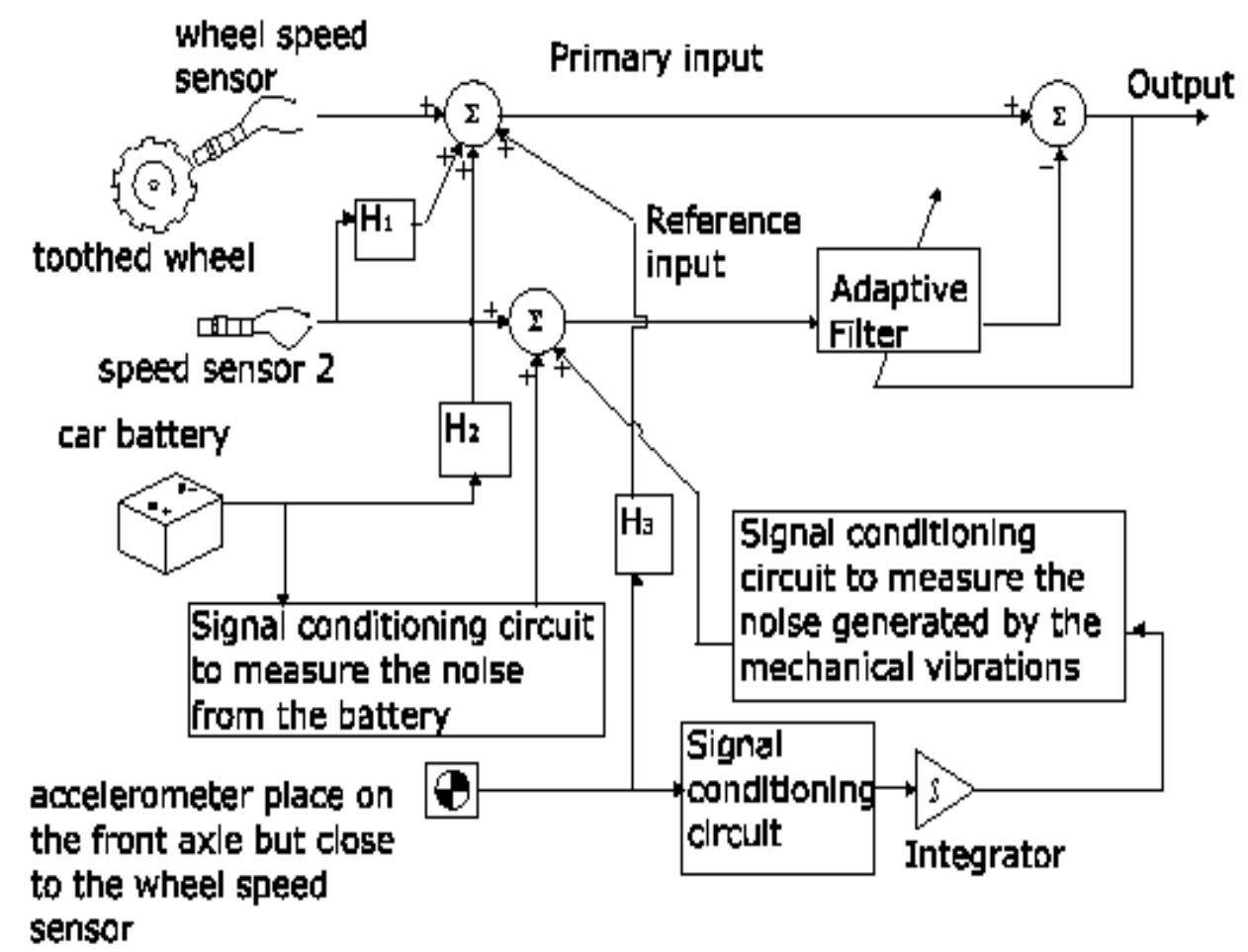

Figure 4. Block diagram of the adaptive noise canceling system.

\subsection{Filtering}

After studying the bandwidth of the relevant signal, a sampling frequency of $10 \mathrm{kHz}$ was chosen. Moreover, the signal treatment was carried out by using a laptop computer and the National Instruments Data Acquisition Card DAQCard-700, both placed in the car under performance tests. Fig. 5 shows the information coming from the wheel speed sensor (i.e., the primary input), and its power spectrum magnitude is shown in Fig. 6. The complete process of data capture and processing ranges from 0 to approximately $42 \mathrm{~km} / \mathrm{h}$ and more than 30 experimental tests were carried out under laboratory conditions.

In addition, the mean-squared error (i.e., the cost function) of the filter is

$$
\mathrm{J}=\frac{1}{2} \mathrm{E}\left[\left.\eta_{\mathrm{R}}(\mathrm{n})\right|^{2}+\left|\beta_{\mathrm{R}}(\mathrm{n})\right|^{2}\right]
$$

where $\mathrm{E}$ is the expectation operator. 


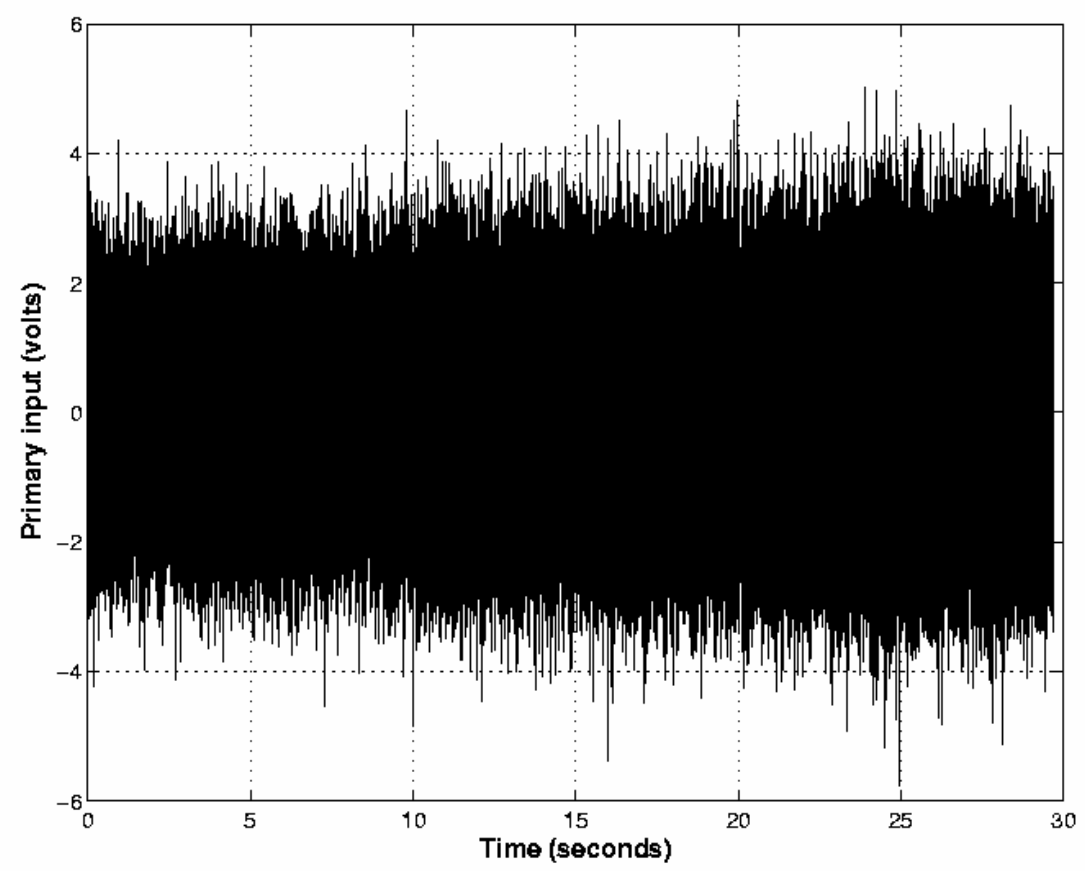

Figure 5. Primary input.

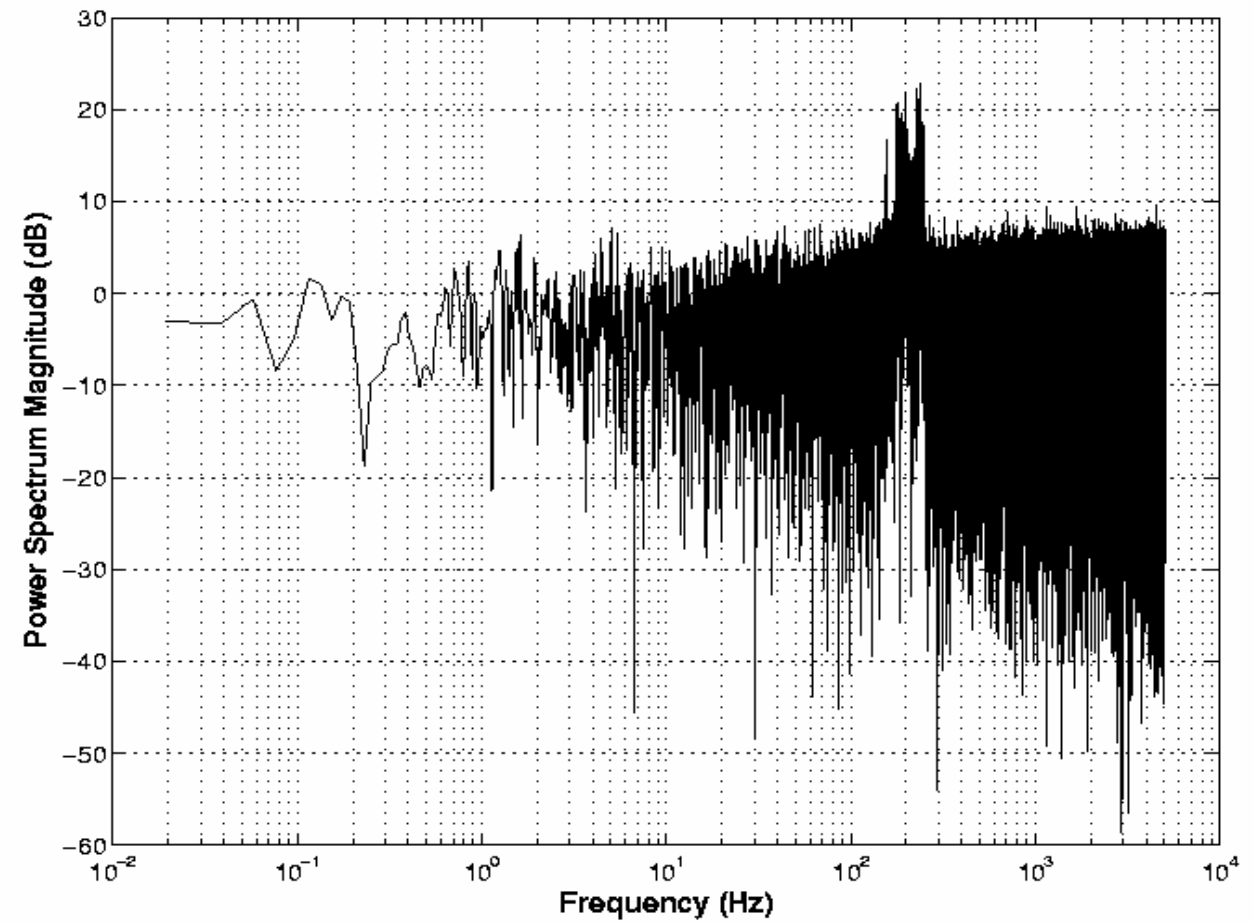

Figure 6. Power spectrum magnitude of the primary input. 
Furthermore, Table 1 shows the normalized ensemble-averaged mean-squared error of the filter over 30 independent trials of the experiment. Such independent trials were carried out in the following way: 3 independent trials were done for each one of the ten possible combinations of the variables shown in Table 1, the length (or number of taps) of the filter $(R+1)$ and the forgetting factor $(\lambda)$.

What is more, the values of $\mathrm{R}$ were chosen in accordance with the idea of implementing an adaptive filter with a small number of adaptive weights. Furthermore, experience tells us that the values of $\lambda$ should be the ones in the range from 0.99 to 1 .

In the case under study, if $\lambda$ is lower than 0.99 , the system is numerically unstable. In short, if $\lambda$ is lower than 0.99 , the system has poor numerical behavior, i.e. it becomes numerically inaccurate, and works with inaccurate values of the forward and backward reflection coefficients (see subsection 4.2). Then, the positive definiteness of the underlying inverse correlation matrix of the input data is lost. Therefore, the system does not converge and its output starts to oscillate in an uncontrollable manner.

Table 1. Normalized ensemble-averaged mean-squared error of the filter for ten combinations of the length of the filter and the forgetting factor.

\begin{tabular}{|c|c|c|}
\hline & \multicolumn{2}{|c|}{$\lambda$} \\
\hline $\mathbf{R}+\mathbf{1}$ & 0.999 & 1 \\
\hline 10 & 0.5536 & 0.5509 \\
\hline 15 & 0.5574 & 0.5406 \\
\hline 20 & 1 & 0.5557 \\
\hline 25 & 0.5626 & 0.7635 \\
\hline 30 & 0.5574 & 0.5611 \\
\hline
\end{tabular}

Here, it is important to point out that one may use a large number of adaptive weights, that is to say a large number of taps of the filter, but doing so could cause problems due to weight-vector noise. In short, a large number of taps could increase the difference between the ensemble-average value of the tap-weight vector and the tap-weight vector (such a difference is called the weight-vector noise), and this increment makes the figures of merit for assessing the tracking capability of the RLS lattice adaptive filter worse. Such figures of merit are the estimation variance and the misadjustment of the adaptive filter.

The previously mentioned problems diminish the detection ability of the main signal due to spurious peaks, which may be confused with the signal of interest. What is more, infinite length of the weight-vector is the ideal solution but in digital signal processing high-order filters increase the computational burden and therefore the speed of the required processor. In addition, high-order filters require increased software complexity, which increases coding and debugging time [55]. For this reason, this paper is focused on finding an estimate of the optimal filter using a small number of taps of the filter.

In accordance with the above statements and the information shown in Table 1, Fig. 7 shows the output of the system, where $\mathrm{R}=14$ and $\lambda=1$, and Fig. 8 shows its power spectrum magnitude.

Here, it is important to point out that the closer $\lambda$ is to 1 , the better the performance of the adaptive filter. Nevertheless, it is not correct the idea that the larger the number of taps of the adaptive filter, the 
better the filter. Therefore, for each specific application, it is suggested that the designer tests the performance of the RLS lattice adaptive filter for several values of $\mathrm{R}$ and $\lambda$ before making his/her final choice.

In this paper, the signal-to-noise ratio (SNR) of the primary input (see Fig. 5 and Fig. 6) is approximately $10 \mathrm{~dB}$, and the SNR at the system output (see Fig. 7 and Fig. 8) is approximately $40 \mathrm{~dB}$. Therefore, a SNR improvement of approximately $30 \mathrm{~dB}$ was achieved, which is a good performance factor for judging the quality of the filter.

In addition, it should be highlighted that the noise that corrupts the relevant signal is so high that the information coming from the wheel speed sensor (see Fig. 5) at near zero speed is completely wrong. However, the system output (see Fig. 7) is satisfactory. The adaptive noise canceller removed a huge amount of noise from the primary input (compare Fig. 6 with Fig. 8).

The experimental results presented in this paper show that the adaptive noise canceller significantly reduced the noise corrupting the relevant information while leaving the important information relatively unchanged from a practical viewpoint.

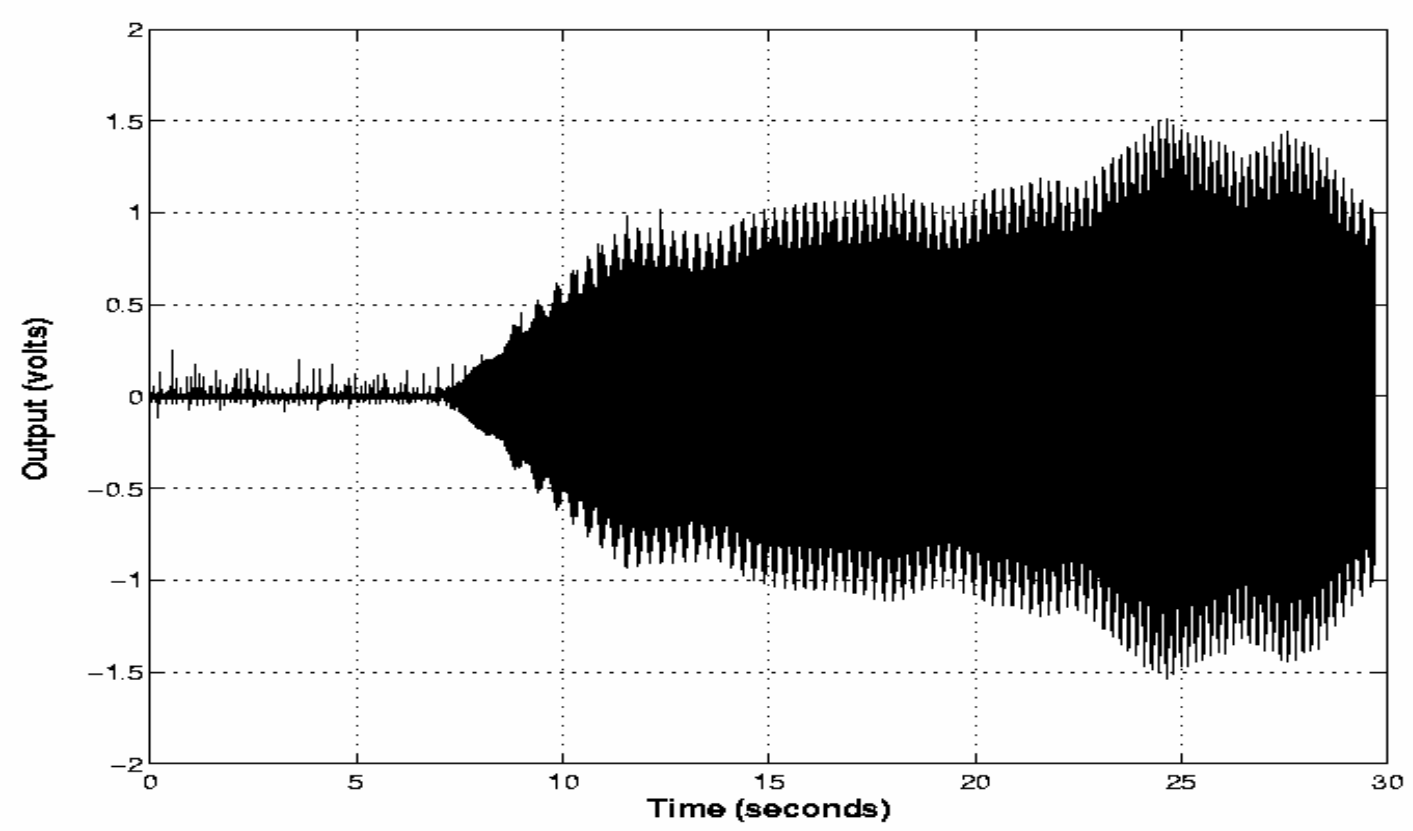

Figure 7. System output.

\section{Conclusions}

To conclude, the effect of the improved sensor signals on the ABS was very positive due to the fact that the results of this research allow the ABS of today's cars to perform much better at the end of the braking process (a safety-related problem).

In short, a RLS lattice adaptive filter was used to improve the performance of a wheel speed sensor placed in a car under performance tests. In addition, a SNR improvement of $30 \mathrm{~dB}$ was achieved, which is a good performance factor for judging the quality of the filter. What is more, the optimal 
system presented in this paper was built by using low-cost components and the system does not need expensive sensors to work satisfactorily, which is a very important factor to be taken into consideration when constructing non-luxury cars.

Furthermore, the results of this paper help the ABS, the traction control system and the electronic stability program, among other electronic systems, to make intelligent driving decisions that can save thousands of lives each year in car accidents. This paper's design method can bridge the gap between intelligent signal processing methods and the design of the intelligent sensors that today's cars need.

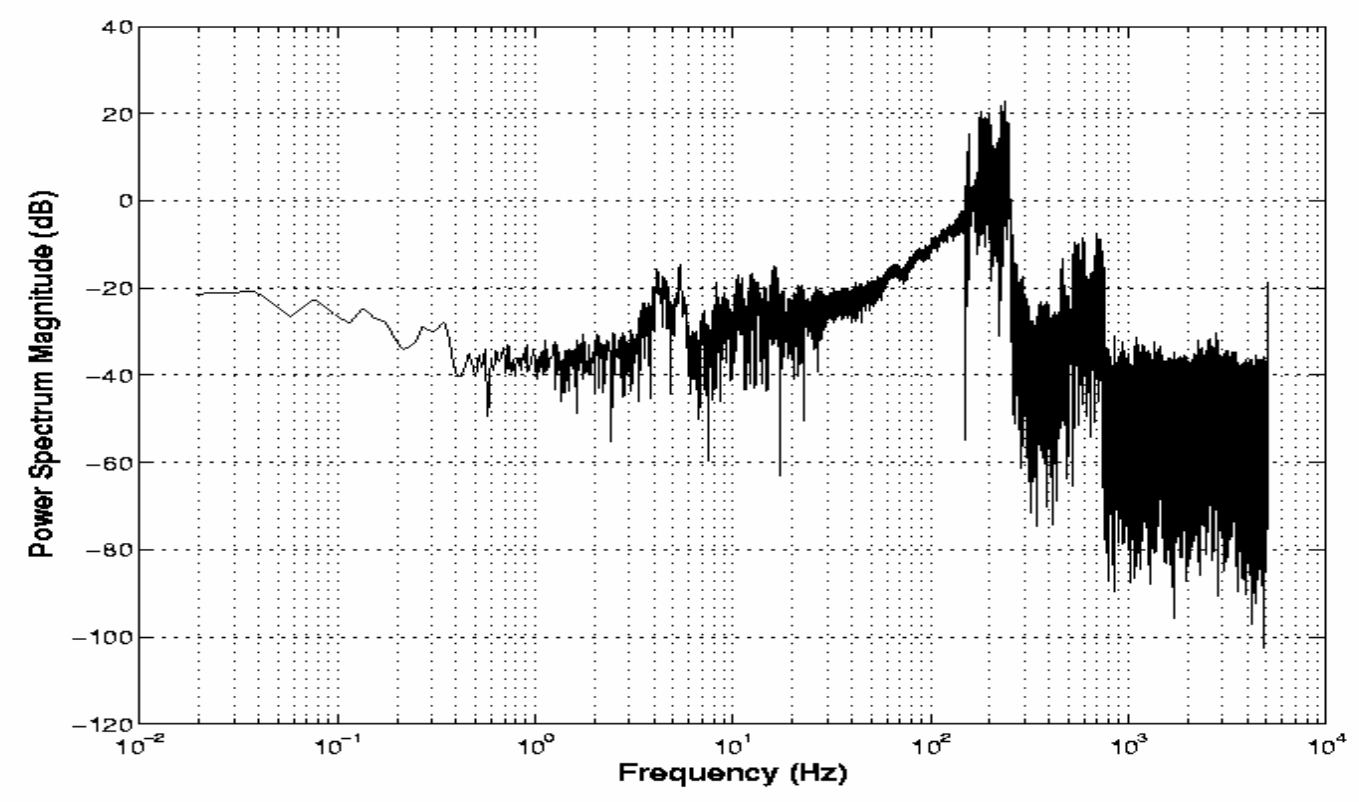

Figure 8. Power spectrum magnitude of the system output.

\section{Acknowledgements}

This work was supported by the Department of Circuits and Systems in the EUIT de Telecomunicacion at the Universidad Politecnica de Madrid, Spain.

\section{References}

1. Microelectromechanical systems advanced materials and fabrication materials. National Academy Press, Washington, D.C., 1997.

2. Cooper, A. R.; Brignell, J. E. Electronic processing of transducer signals: Hall effect as an example. Sensors and Actuators A 1985, 7, 189-198.

3. Ko, W. H.; Fung, C. D. VLSI and intelligent transducers. Sensors and Actuators A 1982, 2, 239250.

4. Middelhoek, S.; Hoogerwerf, A. C. Smart sensors: When and where?. Sensors and Actuators A 1985, 8, 39-48.

5. Giachino, J. M. Smart sensors. Sensors and Actuators A 1986, 10, 239-248. 
6. Middelhoek, S.; French, P. J.; Huising, J. H.; Lian, W. J. Sensors with digital or frequency output. Sensors and Actuators A 1988, 15, 119-133.

7. Holzlein, K.; Larik, J. Silicon magnetic field sensor with frequency output. Sensors and Actuators A 1991, 26, 349-355.

8. Najafi, K. Smart sensors. Journal of Micromechanics and Microengineering 1991, 1, 86-102.

9. Juds, S. M. Toward a definition of smart sensors. Sensors 1991, 2-3.

10. Nihtianov, S.; Minkova, T. Magnetic-field-sensitive device with frequency output. Sensors and Actuators A 1992, 30, 101-104.

11. Ramsden, E. Measuring magnetic fields with fluxgate sensors. Sensors 1994, 87-90.

12. Hernandez, W. Fluxgate magnetometer for magnetic fields in the range 1-100uT. Electronics Letters 1995, 31, 2110-2111.

13. Rodriguez, F.; Trujillo, H.; Hernandez, W. A simple bandgap-type magnetoamplifier. Sensors and Actuators A 1996, 55, 133-137.

14. Hernandez, W. Magnetic-field sensor based on a relaxation oscillator. Sensors and Actuators A 1996, 55, 163-166.

15. Jacobsen, E. Signal conditioning a pressure sensor with a Filed-Programmable Analog Array. Sensors 1997, 81-86.

16. Frank, R. Understanding smart sensors, Second Edition; Artech House, Norwood, MA, 2000.

17. Hernandez, W. Improving the response of an accelerometer by using optimal filtering. Sensors and Actuators A 2001, 88, 198-208.

18. Hernandez, W. Improving the response of several accelerometers used in a car under performance tests by using Kalman filtering. Sensors 2001, 1, 38-52.

19. Hernandez, W. Improving the response of a wheel speed sensor using an adaptive line enhancer. Measurement 2003, 33, 229-240.

20. Hernandez, W. Improving the response of a wheel speed sensor by using frequency-domain adaptive filtering. IEEE Sensors Journal 2003, 3, 404-413.

21. Mörbe, M.; Zwiener, G. Wheel-speed sensors. In Sensors for Automotive Technology; Marek, J., Trah, H. -P., Suzuki, Y., Yokomori, I., Ed.; WILEY-VCH Verlag GmbH \& Co. KgaA, Weinheim 2003, vol. 4, 403-416.

22. Hernandez, W. Improving the response of wheel speed sensors by using robust and optimal signal processing techniques. IEEE International Symposium on Industrial Electronics, ISIE2005, Dubrovnik, Croatia, June 20-23, 2005, 1049-1053.

23. Hernandez, W. Robust multivariable estimation of the relevant information coming from a wheel speed sensor and an accelerometer embedded in a car under performance tests. Sensors 2005, 5, $488-508$.

24. Hernandez, W. Improving the response of a rollover sensor placed in a car under performance tests by using a RLS lattice algorithm. Sensors 2005, 5, $613-632$.

25. Hernandez, W. Wheel speed sensors. In Encyclopedia of Sensor; Grimes, C. A., Dickey, E. C., Pishko, M. V., Ed.; American Scientific Publishers, in press.

26. Gregory, B. A. Introduction to electrical instrumentation and measurement systems, Second Edition; The Macmillan Press Limited, England, 1981 
27. Beckwith, T. G.; Buck, N. L.; Marangoni, R. D. Mechanical measurements, Third Edition; Addison-Wesley, Massachusetts, 1981

28. Norton, H. N. Sensor and analyzer handbook; Prentice-Hall, Englewood Cliffs, New Jersey, 1982.

29. Beckwith, T. G.; Marangoni, R. D.; Lienhard V, J. H. Mechanical measurements, Fifth Edition; Addison-Wesley, New York, 1993.

30. Carr, J. J. Sensors and circuits. Prentice-Hall, Upper Saddle River, New Jersey, 1993.

31. Carr, J. J. Elements of electronic instrumentation and measurements, Third Edition; Prentice-Hall, Upper Saddle River, New Jersey, 1995.

32. Johnson, C. D. Process control instrumentation technology, Fifth Edition; Prentice-Hall, Upper Saddle River, New Jersey, 1997.

33. Edgar, P. Sensors for measurement and control; Longman, London, 1998.

34. Ribbens, W. B. Understanding automotive electronics, Fifth Edition; SAE International, Warrendale, PA, 1998.

35. Webster, J. G. The measurement, instrumentation, and sensors handbook; CRC Press LLC, Boca Raton, Florida, 1999.

36. Pallás-Areny, R.; Webster, J. G. Sensors and signal conditioning, Second Edition; John Wiley \& Sons, New York, 2001.

37. Sinclair, I. Sensors and transducers, Third Edition; Newnes, Butterworth-Heinemann, Linacre House, Jordan Hill, Oxford, 2001.

38. Fleming, W. J. Overview of automotive sensors. IEEE Sensors Journal 2001, 1, 296-308.

39. Wilson, J. S. Sensor technology handbook; Newnes, Elsevier Inc., Linacre House, Jordan Hill, Oxford, UK, 2005.

40. Aparicio, F.; Vera, C.; Díaz, V. Teoría de los vehículos automóviles; Sección de Publicaciones de la ETSII-UPM, Madrid, 1995.

41. Doob, J. L. Stochastic processes; John Wiley \& Sons, New York, 1953.

42. Dynkin, E. B. Markov processes; Springer-Verlag, New York, 1965.

43. Papoulis, A.; Pillai, S. U. Probability, random variables, and stochastic processes, Fourth Edition; McGraw-Hill, New York, 2001.

44. Kalman, R. E. A new approach to linear filtering and prediction problems. Trans. ASME, J. Basic Eng. 1960, 82, 35-45.

45. Kalman, R. E.; Bucy, R. S. New results in linear filtering and prediction theory. Trans. ASME, J. Basic Eng. 1961, 83, 95-107.

46. Kalman, R. E. New Results in Wiener Filtering Theory. Proc. Symp. Eng. Appl. Random Functions Theory and Probability; Bobdanoff, J. L., Kozin, F., Ed.; John Wiley \& Sons, New York, 1963

47. Sorenson, H. W. Least-squares estimation: from Gauss to Kalman. IEEE Spectrum 1970, 7, 63-68.

48. Kailath, T. A view of three decades of linear filtering theory. IEEE Transactions on Information Theory 1974, 20, 146-181.

49. Anderson, B. D.; Moore, J. B. Optimal Filtering; Dover Publications, New York, 2005.

50. Wiener, N. Extrapolation, Interpolation \& Smoothing of Stationary Time Series, The M.I.T. Press, Cambridge, Massachusetts, 1949. 
51. Kolmogorov, A. N; Interpolation and Extrapolation, Bull. de l'académie des sciences de U.S.S.R. 1941, Ser. Math. 5, 3-14.

52. Goodwin, G. S.; Sin, K. S. Adaptive Filtering Prediction and Control; Prentice-Hall, Englewood Cliffs, New Jersey, 1984.

53. Widrow, B.; Stearns, S. D. Adaptive Signal Processing; Prentice-Hall, Englewood Cliffs, New Jersey, 1985.

54. Solo, V.; Kong, X. Adaptive Signal Processing Algorithms; Prentice-Hall, Englewood Cliffs, New Jersey, 1995.

55. Haykin, S. Adaptive Filter Theory, Fourth Edition; Prentice-Hall, Upper Saddle River, New Jersey, 2002.

56. Manolakis, D. G.; Ingle, V. K.; Kogon, S. M. Statistical and adaptive signal processing; Artech House, Norwood, MA, 2005.

57. Pan, C. T.; Plemmons, R. J. Least-squares modifications with inverse factorizations: Parallel implications. Journal of Computational and Applied Mathematics 1989, 27, 109-127.

58. Alexander, S. T. A method for recursive least-squares filtering based upon an inverse QR decomposition. IEEE Transactions on Signal Processing 1993, 41, 20-30.

(C) 2006 by MDPI (http://www.mdpi.net). Reproduction is permitted for noncommercial purposes. 